\title{
Nachweis einer Kuhmilchallergie mit Aminosäureformula
}

\begin{abstract}
- Gastroösophagealer Reflux (GER) ist bei Säuglingen häufig. Jeder zweite ist betroffen. Kommen Komplikationen, wie starke Unruhe, Gedeihstörung oder Ösophagitis, dazu, spricht man von GERD - der gastroösophagealen Reflux-Krankheit, die weiter abgeklärt und behandelt werden muss. Da bei etwa 40\% der Säuglinge eine Kuhmilchallergie die Ursache darstellt, lohnt sich ein Therapieversuch mit Eliminationsdiät, wie PD Dr. Henrik Köhler aus Erlangen berichtete.

Die europäische und die nordamerikanische Gesellschaft für Kindergastroenterologie (ESPGHAN, NASPGHAN) empfehlen in ihren aktuellen Leitlinien bei Kindern mit rezidivierendem Reflux und geringer Gewichtszunahme einen zweiwöchigen Therapieversuch mit extensiv hydrolysierten Formula (eHF) oder Aminosäureformula, um eine Kuhmilchallergie auszuschließen. Das Gleiche gilt für Kinder mit unerklärlichem Schreien. Auch hier kann die Ursache eine Kuhmilchallergie sein, für die es keinen verlässlichen Test zur Vorhersage gibt.
\end{abstract}

Einem Diagnose- und Therapiealgorithmus zufolge, den Köhler vorstellte, erhalten Kinder mit GERD zunächst eHF oder Aminosäureformula. Bei gestillten Kindern ist auf kuhmilchfreie Ernährung der Mutter zu achten. Spricht die Therapie an, wird nach einigen Wochen re-provoziert; bei erneuter Verschlechterung ist die Behandlung mit Formulanahrung mindestens bis zum zwölften Lebensmonat fortzusetzen, um dann erneut kontrolliert zu werden. Spricht die Therapie nicht an, sollten Kinder, die bislang nur eHF erhalten hatten, auf Aminosäureformula wie Neocate ${ }^{\circledR}$ gesetzt werden, die $100 \%$ allergenfrei sind. Bleibt auch dann eine Besserung aus, ist eine Kuhmilchallergie unwahrscheinlich und eine weitere Abklärung angezeigt.

koc

Satellitensymposium „Wenn Essen keinen Spaß mehr macht - Herausforderungen bei der Ernährung von Kindern", anlässlich der 25. Jahrestagung der GPGE. Bonn, 12. Mai 2010. Veranstalter: Pfrimmer Nutricia, Erlangen

\section{Neue Therapiemöglichkeit für Faktor-XIII-Mangel}

— Ein Faktor-XIII-(FXIII-)Mangel ist selten. Um die Betroffenen insbesondere bei milden Formen frühzeitig zu diagnostizieren und einer adäquaten Therapie zuzuführen, sei es wichtig, so Johannes Oldenburg, Direktor des Instituts für Experimentelle Hämatologie und Transfusionsmedizin des Universitätsklinikums Bonn, die Wahrnehmung für dieses Krankheitsbild zu verbessern. Oldenburg fordert mehr Aufmerksamkeit, insbesondere bei unspezifischen Symptomen. Die Behandlung besteht in der Substitution von FXIII-Konzentraten. Als neue Therapieoption wird derzeit das erste rekombinante Präparat in einer Phase-IIIStudie klinisch geprüft.

Ein angeborener FXIII-Mangel kommt relativ selten vor. Leitsymptome des homozygoten FXIII-Mangels sind Nabelschnurund intrakranielle Blutungen. Im Gegensatz dazu sind heterozygote FXIII-Mangel-Patienten bei einer Restaktivität von 40-65\% häufig asymptomatisch oder weisen nur milde Symptome auf, wie etwa leicht erhöhte Blutungsneigung z. B. bei einem operativen Eingriff oder einer Zahnextraktion. Man schätzt, dass etwa 80.000 Patienten in Deutschland betroffen sind.

Die Diagnose ist in der Praxis nicht einfach zu stellen, denn der Defekt wird mit den Routine-Gerinnungsparametern Partielle Thromboplastinzeit (PTT) oder Thromboplastinzeit (TPZ, Quick) nicht erfasst, sondern durch Bestimmung des FXIII-Plasmaspiegels diagnostiziert. Zu der geringen Diagnoserate trägt auch bei, so Oldenburg, dass immer noch die Lehrmeinung vertreten wird, eine FXIII-Aktivität von 10\% sei ausreichend. Kürzlich veröffentlichte Arbeiten zu den milden Formen des FXIIIMangels widersprechen dem jedoch eindeutig.

ch

Fachpressekonferenz ,The Many Faces of Bleeding Disorders“. Bonn, 19. April 2010. Veranstalter: Novo Nordisk, Mainz

\section{Zink ist nicht gleich Zink}

In den westlichen Industrienationen ist Zinkmangel keine Seltenheit - trotz des reichhaltigen Lebensmittelangebotes. Ein Grund ist der rückläufige Fleischverzehr und der stetig wachsende Anteil vegetabiler Kost als Folge veränderter Ernährungsgewohnheiten. Während rotes Fleisch reich an Zink ist, sind Obst und Gemüse eine schlechte Zinkquelle. Ähnliches trifft auf Vollkornprodukte und Hülsenfrüchte zu: Sie weisen einen hohen Gehalt an Phytinsäure auf, die nicht resorbierbare Komplexe mit Zink bildet. Die Wahl des richtigen Zinkpräparates hat entscheidenden Einfluss auf die erfolgreiche Behebung von Zinkmangelzuständen. Zahlreiche tierexperimentelle und klinische Studien belegen, dass die organische Zinkverbindung Zinkhistidin intestinal optimal resorbiert wird: Ihre Resorptionsrate liegt um 30-40\% höher als beispielsweise von Zinksulfat. Zinkhistidin (z. B. Curazink $^{\circledR}$ ) garantiert somit eine maximale Bioverfügbarkeit.

Nach Informationen von STADA, Bad Vilbel

\section{Wachsende Herausforderungen}

Seit der Markteinführung von Medikinet ${ }^{\circledR}$ ist nicht nur die Zahl der behandelten Patienten gestiegen. Die Patienten sind älter geworden und viele von innen befinden sich jetzt in der Pubertät. Im Umgang mit ihren heranwachsenden Patienten haben Ärzte oftmals mit mangelnder Compliance bei der Medikamenteneinnahme zu kämpfen, erläuterte Prof. Michael Huss, Mainz. Huss nannte zwei Gründe dafür, warum die Pubertät für ADHS-Patienten besonders problematisch ist: Zum einen erfahren die Jugendlichen in ihrem Umfeld die Einnahme von Medikamenten als stigmatisierend - und setzen ihre Arzneien eigenmächtig und oftmals heimlich ab. Gleichzeitig verstärken sich in diesem Alter Probleme bei der Impulskontrolle, was den Betroffenen die berufliche Zukunft verbaut und den Einstieg zur Substanzabhängigkeit begünstigen kann. „Man muss vorher einen festen therapeutischen Kontakt zu dem betroffenen Kind aufbauen, dann lässt sich die Zeit der Pubertät besser durchstehen“, lautet der Ratschlag des Kinder- und Jugendpsychiaters.

Nach Informationen von Medice, Iserlohn 